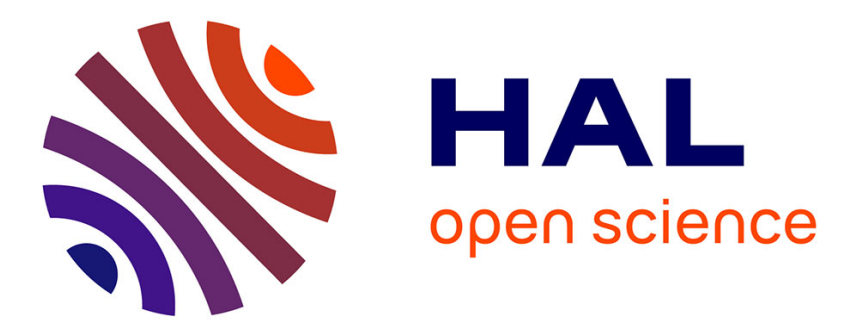

\title{
A self-consistent estimate for linear viscoelastic polycrystals with internal variables inferred from the collocation method
}

Quoc Huy Vu, Renald Brenner, Olivier Castelnau, Hervé Moulinec, Pierre Suquet

\section{To cite this version:}

Quoc Huy Vu, Renald Brenner, Olivier Castelnau, Hervé Moulinec, Pierre Suquet. A self-consistent estimate for linear viscoelastic polycrystals with internal variables inferred from the collocation method. Modelling and Simulation in Materials Science and Engineering, 2012, 20 (2), pp.024003. 10.1088/0965-0393/20/2/024003 . hal-00718324

\section{HAL Id: hal-00718324 \\ https://hal.science/hal-00718324}

Submitted on 16 Jul 2012

HAL is a multi-disciplinary open access archive for the deposit and dissemination of scientific research documents, whether they are published or not. The documents may come from teaching and research institutions in France or abroad, or from public or private research centers.
L'archive ouverte pluridisciplinaire HAL, est destinée au dépôt et à la diffusion de documents scientifiques de niveau recherche, publiés ou non, émanant des établissements d'enseignement et de recherche français ou étrangers, des laboratoires publics ou privés. 


\title{
A self-consistent estimate for linear viscoelastic polycrystals with internal variables inferred from the collocation method
}

\author{
Q. H. Vu ${ }^{1,2}$, R. Brenner ${ }^{1}$, O. Castelnau ${ }^{2}$, H. Moulinec ${ }^{3}$, P. \\ Suquet $^{3}$ \\ ${ }^{1}$ LSPM, CNRS, Université Paris 13, 99 avenue Jean-Baptiste Clément, 93430 \\ Villetaneuse, France \\ 2 PIMM, CNRS, Arts \& Métiers ParisTech, 151 Bd de l'hopital, 75013 Paris, France \\ 3 LMA, CNRS, 31 chemin Joseph Aiguier, 13402 Marseille Cedex 20, France \\ E-mail: olivier.castelnau@ensam.eu
}

\begin{abstract}
The correspondence principle is customarily used with the Laplace-Carson transform technique to tackle the homogenization of linear viscoelastic heterogeneous media. The main drawback of this method lies in the fact that the whole stress and strain histories have to be considered to compute the mechanical response of the material during a given macroscopic loading. Following a remark of Mandel (1966), Ricaud and Masson (2009) have shown the equivalence between the collocation method used to invert Laplace-Carson transforms and an internal variables formulation. In the present article, this new method is developed for the case of polycrystalline materials with general anisotropic properties for local and macroscopic behaviors. Applications are provided for the case of constitutive relations accounting for glide of dislocations on particular slip systems. It is shown that the method yields accurate results that perfectly match the standard collocation method and reference full-field results obtained with a FFT numerical scheme. The formulation is then extended to the case of time and strain dependent viscous properties, leading to the Incremental Collocation Method (ICM) that can be solved efficiently by a step-by-step procedure. Specifically, the introduction of isotropic and kinematic hardening at the slip system scale is considered.
\end{abstract}




\section{Introduction}

The principal issue for the homogenization of linear viscoelastic heterogeneous media is due to memory effects. Owing to the dependence of the local strain-rate on both local stress (viscous response) and stress-rate (elastic response), standard homogenization methods developed for elasticity or viscoplasticity do not apply directly. In particular, the whole stress and strain histories have to be considered for determining the mechanical response at a given time. From the practical point of view of numerical applications, this property requires storing the stress and strain in each mechanical phase for the whole loading path. This can be quite cumbersome especially for polycrystals which are heterogeneous media containing a large number (typically a few thousands) of constituent phases (i.e. crystalline orientations).

Approximate solutions based on properly chosen internal variables have been proposed, e.g. see $[1,2,3,4]$. Internal variables aim to keep track of the whole stress/strain histories and to summarize their effect on the material behavior. Since their number is limited, the amount of information required to predict the next time step is by far smaller than with hereditary approaches considering full memory effects (at the expense of a lesser accuracy). An alternative internal variable method, based on an incremental variational principle, has been proposed by $[5,6]$. By contrast with previous approaches, this method makes use of the recent developments for the homogenization of nonlinear composites which rely on the use of both first and second moments of the mechanical fields to define a linear comparison composite (LCC) $[7,8,9]$ and effective internal variables per phase.

The homogenization problem can be solved exactly by making use of the correspondence principle [10]. Taking the Laplace-Carson (LC) transform of the problem, the linear viscoelastic behavior is transformed into a symbolic linear elastic one in the LC space. Any linear homogenization model can thus be applied to the fictitious elastic heterogeneous material. Then, inverse LC transforms must be carried out to obtain the viscoelastic effective property in the cartesian space. Except for few simple cases for which this inversion can be performed exactly [11, 12] and besides a simple approximate solution (direct method) which is only accurate for specific loading paths such as creep [13, 14, 15], numerical inversions are generally required. For this goal, approaches relying on Dirichlet series expansion of the original time functions have been proposed, e.g. [13, 16, 17, 18]. Among them, the so-called collocation method [13] has provided very good results for the homogenization of polycrystals [19, 20, 21].

Recently, Ricaud and Masson [22] have made use of a remark of [10] showing that an internal variable formulation arises naturally from the collocation method. They illustrated the potentiality of this new method for the case of a two-phase composite with isotropic phases. The main attractive feature of this formulation is to keep the accuracy of integral approaches while preserving the flexibility of an internal variable approach.

The present study develops one step further the approach of Ricaud and Masson 
[22]. We consider $n$-phase polycrystalline materials with general anisotropic properties for local and macroscopic behaviors and applications are provided for constitutive relations accounting for glide of dislocations on particular slip systems. We recall in section 2 the basic equations for the homogenization of thermoviscoelastic heterogeneous media and it is shown in section 3 how internal variables come out naturally from the collocation method, for both stress and strain formulations. Applications are provided for a 2-D isotropic polycrystal submitted to (i) creep loading with stress discontinuities and (ii) complex loading path with strain harmonic loading. In section 4, the method is extended to time- and strain- dependent viscous properties (resulting from isotropic and kinematic hardening on slip systems), which can be solved with a step-by-step procedure. The results obtained for a loading path containing a harmonic part are compared to reference solutions obtained by the FFT full-field numerical approach of [23].

\section{Homogenization of linear thermoviscoelastic heterogeneous media}

\subsection{Constitutive equation for local behavior}

According to the Boltzmann superposition principle, the local stress (resp. strain) response of a heterogeneous linear thermoviscoelastic medium can be expressed as a Stieltjes convolution of a viscoelastic stiffness (resp. compliances) tensor with strain (resp. stress), with the addition of thermal stress (resp. strain), that is

$$
\begin{aligned}
& \boldsymbol{\sigma}(\mathbf{x}, t)=[\mathbf{C} \star \boldsymbol{\varepsilon}](\mathbf{x}, t)+\boldsymbol{\sigma}_{0}(\mathbf{x}, t) \\
& \boldsymbol{\varepsilon}(\mathbf{x}, t)=[\mathbf{S} \star \boldsymbol{\sigma}](\mathbf{x}, t)+\boldsymbol{\varepsilon}_{0}(\mathbf{x}, t)
\end{aligned}
$$

with $\mathbf{x}$ and $t$ the space and time variables, $\sigma$ and $\varepsilon$ stress and strain tensors, $\star$ the Stieltjes convolution product, $\mathbf{C}$ the viscoelastic stiffness tensor (i.e. relaxation function), and $\mathbf{S}$ the viscoelastic compliance (i.e. creep function). Stieltjes convolutions being defined as the time derivative of Riemann convolutions, the constitutive law is obtained by considering the superimposition of infinitesimal and finite strain increments, $\mathrm{d} \varepsilon$ and $[\varepsilon]$,

$$
\begin{aligned}
\boldsymbol{\sigma}(\mathbf{x}, t) & =\frac{\mathrm{d}}{\mathrm{d} t}\left[\int_{0}^{t} \mathbf{C}(\mathbf{x}, t-u): \varepsilon(u) \mathrm{d} u\right]+\boldsymbol{\sigma}_{0}(\mathbf{x}, t) \\
& =\int_{0}^{t} \mathbf{C}(\mathbf{x}, t-u): \dot{\varepsilon}(u) \mathrm{d} u+\sum_{d} \mathbf{C}\left(\mathbf{x}, t-t_{d}\right):[\varepsilon]_{d}+\boldsymbol{\sigma}_{0}(\mathbf{x}, t) .
\end{aligned}
$$

In this expression, strain discontinuities $[\varepsilon]_{d}$ are considered only for times $t_{d}<t$. Alternatively, the constitutive behaviour reads

$$
\begin{aligned}
\varepsilon(\mathbf{x}, t) & =\frac{\mathrm{d}}{\mathrm{d} t}\left[\int_{0}^{t} \mathbf{S}(\mathbf{x}, t-u): \boldsymbol{\sigma}(u) \mathrm{d} u\right]+\varepsilon_{0}(\mathbf{x}, t) \\
& =\int_{0}^{t} \mathbf{S}(\mathbf{x}, t-u): \dot{\boldsymbol{\sigma}}(u) \mathrm{d} u+\sum_{d} \mathbf{S}\left(\mathbf{x}, t-t_{d}\right):[\boldsymbol{\sigma}]_{d}+\varepsilon_{0}(\mathbf{x}, t)
\end{aligned}
$$

with possible stress discontinuities $[\boldsymbol{\sigma}]_{d}$ at times $t_{d}<t$. 
The creep function of a Maxwell viscoelastic behavior with general anisotropy reads

$$
\mathbf{S}(\mathbf{x}, t)=\mathbf{S}^{e}(\mathbf{x})+\mathbf{S}^{v}(\mathbf{x}) t
$$

with $\mathbf{S}^{e}$ and $\mathbf{S}^{v}$ the elastic and viscous compliances tensors. By definition, the relaxation function $\mathbf{C}(\mathbf{x}, t)$ obeys $\mathbf{C} \star \mathbf{S}=\mathbf{I}$ (I is the identity tensor) but, unlike the creep function, its analytical expression depends on the class of symmetry (Appendix A).

\subsection{Effective behaviour}

We consider the case of a polycrystal made of $R$ mechanical phases (i.e. $R$ crystalline orientations) with $\chi_{r}$ the characteristic function of phase $r$ and $c_{r}$ its volume fraction. The local thermoviscoelastic behavior is maxwellian and uniform in each phase, so that tensors $\mathbf{C}^{e}, \mathbf{C}^{v}, \mathbf{S}^{e}, \mathbf{S}^{v}, \mathbf{C}, \mathbf{S}, \boldsymbol{\sigma}_{0}, \boldsymbol{\varepsilon}_{0}$ are also uniform per phase, and denoted $\mathbf{C}_{r}^{e}, \mathbf{C}_{r}^{v}$, $\mathbf{S}_{r}^{e}, \mathbf{S}_{r}^{v}, \mathbf{C}_{r}, \mathbf{S}_{r}, \boldsymbol{\sigma}_{0 r}$, and $\varepsilon_{0 r}(t)$. Thus

$$
\begin{array}{rlrl}
\mathbf{C}(\mathbf{x}, t) & =\sum_{r} \chi_{r}(\mathbf{x}) \mathbf{C}_{r}(t), & \boldsymbol{\sigma}_{0}(\mathbf{x}, t) & =\sum_{r} \chi_{r}(\mathbf{x}) \boldsymbol{\sigma}_{0 r}(t) \\
\mathbf{S}(\mathbf{x}, t)=\sum_{r} \chi_{r}(\mathbf{x}) \mathbf{S}_{r}(t), & \varepsilon_{0}(\mathbf{x}, t) & =\sum_{r} \chi_{r}(\mathbf{x}) \varepsilon_{0 r}(t)
\end{array}
$$

with $\mathbf{S}_{r}(t)=\mathbf{S}_{r}^{e}+\mathbf{S}_{r}^{v} t$ and $\mathbf{C}_{r} \star \mathbf{S}_{r}=\mathbf{I}$. The average stress and strain (denoted $<.>_{r}$ ) within phase $r$ obey the constitutive relation (1)

$$
\begin{aligned}
\langle\boldsymbol{\sigma}\rangle_{r}(t) & =\left[\mathbf{C}_{r} \star\langle\boldsymbol{\varepsilon}\rangle_{r}\right](t)+\boldsymbol{\sigma}_{0 r}(t) \\
\langle\boldsymbol{\varepsilon}\rangle_{r}(t) & =\left[\mathbf{S}_{r} \star\langle\boldsymbol{\sigma}\rangle_{r}\right](t)+\boldsymbol{\varepsilon}_{0 r}(t)
\end{aligned}
$$

These phase average fields are linked to macroscopic ones by phase average stress and strain localization tensors $\mathbf{B}_{r}(t)$ and $\mathbf{A}_{r}(t) \ddagger$

$$
\begin{aligned}
& \langle\boldsymbol{\sigma}\rangle_{r}(t)=\left[\mathbf{B}_{r} \star \overline{\boldsymbol{\sigma}}\right](t)+\left\langle\boldsymbol{\sigma}_{\text {res }}\right\rangle_{r}(t) \\
& \langle\boldsymbol{\varepsilon}\rangle_{r}(t)=\left[\mathbf{A}_{r} \star \overline{\boldsymbol{\varepsilon}}\right](t)+\left\langle\varepsilon_{\text {res }}\right\rangle_{r}(t)
\end{aligned}
$$

with $\overline{\boldsymbol{\sigma}}$ and $\overline{\boldsymbol{\varepsilon}}$ the overall (macroscopic) stress and strain $(\overline{\boldsymbol{\sigma}}=<\boldsymbol{\sigma}\rangle, \overline{\boldsymbol{\varepsilon}}=<\boldsymbol{\varepsilon}>$, with $<$. $>$ the average over the polycrystal volume). In $(7),\left\langle\varepsilon_{\text {res }}\right\rangle_{r}$ and $\left\langle\boldsymbol{\sigma}_{\text {res }}\right\rangle_{r}$ are the phase average residual stress and strain defined by

$$
\begin{aligned}
& \left\langle\boldsymbol{\sigma}_{\text {res }}\right\rangle_{r}(t)=\left[\mathbf{D}_{r}(t) \star\left(\widetilde{\boldsymbol{\varepsilon}}_{0}-\boldsymbol{\varepsilon}_{0 r}\right)\right](t) \\
& \left\langle\varepsilon_{\text {res }}\right\rangle_{r}(t)=\left[\mathbf{E}_{r}(t) \star\left(\widetilde{\boldsymbol{\sigma}}_{0}-\boldsymbol{\sigma}_{0 r}\right)\right](t)
\end{aligned}
$$

where $\mathbf{D}_{r}(t)$ and $\mathbf{E}_{r}(t)$ are respectively eigenstrain and eigenstress average influence tensors. The macroscopic behavior is given by the effective (denoted ?) relaxation function, creep function, thermal stress, and stress-free strain, given by

$$
\begin{array}{ll}
\widetilde{\mathbf{C}}(t)=\left\langle\mathbf{C}_{r} \star \mathbf{A}_{r}\right\rangle(t), \quad \widetilde{\boldsymbol{\sigma}}_{0}(t)=\left\langle{ }^{\mathrm{T}} \mathbf{A}_{r} \star \boldsymbol{\sigma}_{0 r}\right\rangle(t), \\
\widetilde{\mathbf{S}}(t)=\left\langle\mathbf{S}_{r} \star \mathbf{B}_{r}\right\rangle(t), \quad \widetilde{\boldsymbol{\varepsilon}}_{0}(t)=\left\langle{ }^{\mathrm{T}} \mathbf{B}_{r} \star \boldsymbol{\varepsilon}_{0 r}\right\rangle(t) .
\end{array}
$$

This homogenization problem can be solved by making use of the correspondence principle [10]. Taking the LC transforms of previous equations, Stieljes convolution 
products are transformed into simple scalar products, and therefore the original thermoviscoelastic homogenization problem is transformed into a symbolic thermoelastic homogenization problem for which standard homogenization techniques apply. Let $f^{*}$ denotes the LC transform of function $f, f^{*}(p)=p \int_{0}^{\infty} f(t) e^{-p t} d t$ with $p$ the complex variable. The symbolic thermoelastic behavior thus reads

$$
\begin{array}{ll}
\widetilde{\mathbf{C}}^{*}(p)=\left\langle\mathbf{C}_{r}^{*}(p): \mathbf{A}_{r}^{*}(p)\right\rangle, & \widetilde{\boldsymbol{\sigma}}_{0}^{*}(p)=\left\langle{ }^{\mathrm{T}} \mathbf{A}_{r}^{*}(p): \boldsymbol{\sigma}_{0 r}^{*}(p)\right\rangle \\
\widetilde{\mathbf{S}}^{*}(p)=\left\langle\mathbf{S}_{r}^{*}(p): \mathbf{B}_{r}^{*}(p)\right\rangle, & \widetilde{\boldsymbol{\varepsilon}}_{0}^{*}(p)=\left\langle{ }^{\mathrm{T}} \mathbf{B}_{r}^{*}(p): \boldsymbol{\varepsilon}_{0 r}^{*}(p)\right\rangle
\end{array}
$$

with $\mathbf{S}_{r}^{*}, \mathbf{C}_{r}^{*}, \varepsilon_{0 r}^{*}$ and $\boldsymbol{\sigma}_{0 r}^{*}$ symbolic thermoelastic property tensors. Once the homogenization problem has been solved in the LC space, the solution has to be inverse transformed back to the original time space. This inversion constitutes the main difficulty for thermoviscoelastic homogenization. In this work, use will be made of the collocation method [13]. Following [22], it will be shown that an internal variables formulation can be naturally derived from this inversion procedure, without additional assumptions. It is also pointed out that this feature is not restricted to this specific numerical inversion method.

\section{A formulation based on internal variables inferred from the collocation method}

\subsection{The collocation method}

It is assumed that the considered polycrystal is subjected to a given derivable stress loading path $\overline{\boldsymbol{\sigma}}(u), u \in[0 ; t]$ with additional discontinuities (i.e. stress jumps) $[\overline{\boldsymbol{\sigma}}]_{d}$ at times $t_{d}$, and initial conditions $\boldsymbol{\sigma}(\mathbf{x}, 0)=\overline{\boldsymbol{\sigma}}(0)=\mathbf{0} \forall(\mathbf{x})$. The overall polycrystal response $\bar{\varepsilon}(t)$ reads

$$
\overline{\boldsymbol{\varepsilon}}(t)=\int_{0}^{t} \widetilde{\mathbf{S}}(t-u): \dot{\overline{\boldsymbol{\sigma}}}(u) \mathrm{d} u+\sum_{d} \widetilde{\mathbf{S}}\left(t-t_{d}\right):[\overline{\boldsymbol{\sigma}}]_{d}+\widetilde{\boldsymbol{\varepsilon}}_{0}(t) .
$$

The collocation method is based on an approximation of the effective creep function $\widetilde{\mathbf{S}}$ by a Dirichlet serie $\widetilde{\mathbf{S}}^{a p}$

$$
\widetilde{\mathbf{S}}(t) \approx \widetilde{\mathbf{S}}^{a p}(t)=\widetilde{\mathbf{S}}^{e}+\widetilde{\mathbf{S}}^{v} t+\sum_{s=1}^{S_{c}} \boldsymbol{S}_{\tau_{s}}\left(1-e^{\frac{-t}{\tau_{s}}}\right)
$$

which LC transform reads

$$
\widetilde{\mathbf{S}}^{a p^{*}}(p)=\widetilde{\mathbf{S}}^{e}+\frac{1}{p} \widetilde{\mathbf{S}}^{v}+\sum_{s=1}^{S_{c}} \boldsymbol{S}_{\tau_{s}} \frac{1}{1+\tau_{s} p} .
$$

The $S_{c}$ collocation times $\tau_{s}$ can be chosen optimally as in [24], but here they are supposed to be determined a priori. Equation (13) defines a system of $S_{c}$ linear equations, in which the $S_{c}$ unknown tensors $\boldsymbol{S}_{\tau_{s}}$ are determined from the purely elastic and purely viscous end member solutions (that can be solved independently), and from the symbolic response $\widetilde{\mathbf{S}}^{a p^{*}}(p)$ computed at $S_{c}$ collocation times $p=1 / \tau_{s}$. The effective strain response can then be obtained analytically with relation (11) for any loading path. This method has been used for polycrystalline materials e.g. in [19, 20, 21]. 


\subsection{Stress formulation}

Alternatively, the macroscopic strain can be obtained by substituting $\widetilde{\boldsymbol{S}}(t)$ in (11) by its approximation $\widetilde{\mathbf{S}}^{a p}(t)$. Integrating by part and using condition $\overline{\boldsymbol{\varepsilon}}(0)=\mathbf{0}$ leads to

$$
\begin{aligned}
\overline{\boldsymbol{\varepsilon}}(t)=\widetilde{\boldsymbol{S}}^{e}:\left(\overline{\boldsymbol{\sigma}}(t)+\sum_{d}[\overline{\boldsymbol{\sigma}}]_{d}\right)+\widetilde{\boldsymbol{S}}^{v}:\left(\boldsymbol{\xi}(t)+\sum_{d}\left(t-t_{d}\right)[\overline{\boldsymbol{\sigma}}]_{d}\right)+ \\
\sum_{s=1}^{S_{c}} \boldsymbol{S}_{\tau_{s}}:\left(\boldsymbol{\beta}_{\tau_{s}}(t)+\sum_{d}\left(1-e^{\frac{-\left(t-t_{d}\right)}{\tau_{s}}}\right)[\overline{\boldsymbol{\sigma}}]_{d}\right)+\widetilde{\boldsymbol{\varepsilon}}_{0}(t)
\end{aligned}
$$

where two tensorial internal variables $\boldsymbol{\xi}(t)$ and $\boldsymbol{\beta}_{\tau_{s}}(t)$ arise naturally. They only depend on the macroscopic stress path

$$
\boldsymbol{\beta}_{\tau_{s}}(t)=\frac{1}{\tau_{s}} e^{\frac{-t}{\tau_{s}}} \int_{0}^{t} e^{\frac{u}{\tau_{s}}} \overline{\boldsymbol{\sigma}}(u) \mathrm{d} u, \quad \boldsymbol{\xi}(t)=\int_{0}^{t} \overline{\boldsymbol{\sigma}}(u) \mathrm{d} u,
$$

and are solution of the following differential equations

$$
\dot{\boldsymbol{\beta}}_{\tau_{s}}(t)+\frac{1}{\tau_{s}} \boldsymbol{\beta}_{\tau_{s}}(t)=\frac{1}{\tau_{s}} \overline{\boldsymbol{\sigma}}(t), \quad \dot{\boldsymbol{\xi}}(t)=\overline{\boldsymbol{\sigma}}(t)
$$

with $\boldsymbol{\beta}_{\tau_{s}}(0)=\mathbf{0}, \boldsymbol{\xi}(0)=\mathbf{0}$. Therefore $\boldsymbol{\xi}$ and $\boldsymbol{\beta}_{\tau_{s}}$ are macroscopic variables. Remark that there is a single $\boldsymbol{\xi}$ but as many $\boldsymbol{\beta}_{\tau_{s}}$ as collocation times. It is also worth noting that equations (14-16) could be written alternatively by incorporating stress discontinuities $[\overline{\boldsymbol{\sigma}}]_{d}$ into the definitions of $\boldsymbol{\xi}$ and $\boldsymbol{\beta}_{\tau_{s}}$. If the overall polycrystal loading is performed in such a way that $\overline{\boldsymbol{\varepsilon}}(t)$ is prescribed and $\overline{\boldsymbol{\sigma}}(t)$ is the wanted response, then $\overline{\boldsymbol{\sigma}}(t)$ can be replaced in the above equations (16) by its expression derived from (14). The effective thermal strain $\widetilde{\varepsilon}_{0}(t)$ can be expressed by approximating the phase average stress concentration tensors by a Dirichlet serie, as in [21]

$$
\mathbf{B}_{r}(t) \approx \mathbf{B}_{r}^{a p}(t)=\mathbf{B}_{r}^{v}+\sum_{s=1}^{S_{c}} \mathbf{B}_{r_{\tau_{s}}} e^{\frac{-t}{\tau_{s}}}
$$

leading to

$$
\mathbf{B}_{r}^{a p^{*}}(p)=\mathbf{B}_{r}^{v}+\sum_{s=1}^{S_{c}} \mathbf{B}_{r \tau_{s}} \frac{\tau_{s} p}{1+\tau_{s} p}
$$

where $\boldsymbol{B}_{r}^{v}$ denotes stress concentration tensors for the purely viscous behavior. Tensors $\mathbf{B}_{r \tau_{s}}$ can be easily determined from the knowledge of $\mathbf{B}_{r}^{a p^{*}}$ at collocation times $p=1 / \tau_{s}$, and satisfy $\mathbf{B}_{r}^{v}+\sum_{s=1}^{Q} \mathbf{B}_{r \tau_{s}}=\mathbf{B}_{r}^{e}$ with $\mathbf{B}_{r}^{e}$ the stress concentration tensor for the purely elastic behavior. Using this approximation with the initial condition $\boldsymbol{\varepsilon}_{0 r}(0)=\mathbf{0}$, relation (9d) becomes

$$
\begin{aligned}
\widetilde{\boldsymbol{\varepsilon}}_{0}(t)=\left\langle{ }^{\mathrm{T}} \mathbf{B}_{r}^{e}: \boldsymbol{\varepsilon}_{0 r}(t)+{ }^{\mathrm{T}} \mathbf{B}_{r}^{v}: \sum_{d}\left[\boldsymbol{\varepsilon}_{0 r}\right]_{d}\right\rangle+ \\
\\
\left\langle\sum_{s}{ }^{\mathrm{T}} \mathbf{B}_{r \tau_{s}}:\left(\sum_{d} e^{\frac{-\left(t-t_{d}\right)}{\tau_{s}}}\left[\boldsymbol{\varepsilon}_{0 r}\right]_{d}-\boldsymbol{\eta}_{r \tau_{s}}(t)\right)\right\rangle
\end{aligned}
$$


with the new internal variable $\boldsymbol{\eta}_{r_{s}}$ depending only on the thermal stress-free strain and satisfying

$$
\dot{\boldsymbol{\eta}}_{r_{\tau_{s}}}(t)+\frac{1}{\tau_{s}} \boldsymbol{\eta}_{r_{\tau_{s}}}(t)=\frac{1}{\tau_{s}} \boldsymbol{\varepsilon}_{0 r}(t), \quad \boldsymbol{\eta}_{\tau_{\tau_{s}}}(0)=\mathbf{0} .
$$

Hence, $\boldsymbol{\eta}_{r_{\tau_{s}}}$ is a local variable, and it is worth noting that it is homogeneous per phase due to the homogeneity of $\varepsilon_{0 r}$. It is stressed that the three internal variables determined so far, namely $\boldsymbol{\xi}, \boldsymbol{\beta}_{\tau_{s}}$, and $\boldsymbol{\eta}_{r_{\tau_{s}}}$, can be calculated in advance as far as the macroscopic stress and thermal loadings are known and provided the necessary collocation times $\tau_{s}$ have been fixed. An important consequence of these developments is that the integral expression (11) for the thermoviscoelastic effective behavior has been replaced by the internal variable formulation given by (14) and (19) that can be advantageously solved by means of an incremental numerical procedure.

Similarly, the phase average stress defined by the integral equation (7) can be expressed with respect to internal variables, using the approximation (17) and assuming a similar form for the eigenstrain influence tensors $\mathbf{D}_{r}(t)$

$$
\mathbf{D}_{r}(t) \approx \mathbf{D}_{r}^{a p}(t)=\mathbf{D}_{r}^{v}+\sum_{s=1}^{S_{c}} \mathbf{D}_{r \tau_{s}} e^{\frac{-t}{\tau_{s}}}
$$

This leads to

$$
\begin{aligned}
\langle\boldsymbol{\sigma}\rangle_{r}(t)=\mathbf{B}_{r}^{e}: \overline{\boldsymbol{\sigma}}(t)+\mathbf{B}_{r}^{v}: \sum_{d}[\overline{\boldsymbol{\sigma}}]_{d}+ \\
\sum_{s} \mathbf{B}_{r_{\tau_{s}}}:\left(\sum_{d} e^{\frac{-\left(t-t_{d}\right)}{\tau_{s}}}[\overline{\boldsymbol{\sigma}}]_{d}-\boldsymbol{\beta}_{\tau_{s}}(t)\right)+\left\langle\boldsymbol{\sigma}_{\mathrm{res}}\right\rangle_{r}(t),
\end{aligned}
$$

$$
\begin{aligned}
\left\langle\boldsymbol{\sigma}_{\mathrm{res}}\right\rangle_{r}(t)= & \mathbf{D}_{r}^{e}:\left(\widetilde{\boldsymbol{\varepsilon}}_{0}-\boldsymbol{\varepsilon}_{0 r}\right)(t)+\mathbf{D}_{r}^{v}: \sum_{d}\left[\widetilde{\boldsymbol{\varepsilon}}_{0}-\boldsymbol{\varepsilon}_{0 r}\right]_{d}+ \\
& \sum_{s} \mathbf{D}_{r \tau_{s}}:\left(\sum_{d} e^{\frac{-\left(t-t_{d}\right)}{\tau_{s}}}\left[\widetilde{\boldsymbol{\varepsilon}}_{0}-\boldsymbol{\varepsilon}_{0 r}\right]_{d}-\boldsymbol{\lambda}_{r_{\tau_{s}}}(t)\right)
\end{aligned}
$$

with the new local internal variable $\boldsymbol{\lambda}_{r \tau_{s}}$ verifying

$$
\dot{\boldsymbol{\lambda}_{r \tau_{s}}}(t)+\frac{1}{\tau_{s}} \boldsymbol{\lambda}_{r \tau_{s}}(t)=\frac{1}{\tau_{s}}\left(\widetilde{\boldsymbol{\varepsilon}}_{0}-\boldsymbol{\varepsilon}_{0 r}\right)(t), \quad \boldsymbol{\lambda}_{r \tau_{s}}(0)=\mathbf{0}
$$

The phase average strain $\langle\varepsilon\rangle_{r}(t)$ can be eventually computed by solving incrementally the local constitutive thermoviscoelastic relation.

\subsection{Strain formulation}

We now consider that the polycrystal is subjected to a given derivable loading path $\bar{\varepsilon}(u), u \in[0 ; t]$ with additional discontinuities $[\bar{\varepsilon}]_{d}$ at times $t_{d}$ and initial conditions $\varepsilon(\mathbf{x}, 0)=\bar{\varepsilon}(0)=\mathbf{0} \forall(\mathbf{x})$. The stress response $\overline{\boldsymbol{\sigma}}(t)$ obtained by the strain (or dual) formulation reads

$$
\overline{\boldsymbol{\sigma}}(t)=\int_{0}^{t} \widetilde{\mathbf{C}}(t-u): \dot{\bar{\varepsilon}}(u) \mathrm{d} u+\sum_{d} \widetilde{\mathbf{C}}\left(t-t_{d}\right):[\overline{\boldsymbol{\varepsilon}}]_{d}+\widetilde{\boldsymbol{\sigma}}_{0}(t) .
$$


Approximating the effective relaxation function by a Dirichlet series in a form consistent with (12)

$$
\widetilde{\boldsymbol{C}}(t) \approx \widetilde{\boldsymbol{C}}^{a p}(t)=\sum_{s=1}^{S_{c}} \boldsymbol{C}_{\tau_{s}} e^{\frac{-t}{\tau_{s}}}, \quad \sum_{s=1}^{S_{c}} \boldsymbol{C}_{\tau_{s}}=\widetilde{\boldsymbol{C}}^{e}
$$

the macroscopic stress reads

$$
\overline{\boldsymbol{\sigma}}(t)=\widetilde{\boldsymbol{C}}^{e}: \overline{\boldsymbol{\varepsilon}}(t)-\sum_{s=1}^{S_{c}} \boldsymbol{C}_{\tau_{s}}:\left(\boldsymbol{\alpha}_{\tau_{s}}(t)-\sum_{d} e^{\frac{-\left(t-t_{d}\right)}{\tau_{s}}}[\overline{\boldsymbol{\varepsilon}}]_{d}\right)+\widetilde{\boldsymbol{\sigma}}_{0}(t)
$$

with the macroscopic internal variable $\boldsymbol{\alpha}_{\tau_{s}}$ verifying

$$
\dot{\boldsymbol{\alpha}}_{\tau_{s}}(t)+\frac{1}{\tau_{s}} \boldsymbol{\alpha}_{\tau_{s}}(t)=\frac{1}{\tau_{s}} \overline{\boldsymbol{\varepsilon}}(t), \quad \boldsymbol{\alpha}_{\tau_{s}}(0)=\mathbf{0} .
$$

The effective thermal stress $\widetilde{\boldsymbol{\sigma}}_{0}(t)$ can be expressed by using the following approximation for the average strain concentration tensors

$$
\mathbf{A}_{r}(t) \approx \mathbf{A}_{r}^{a p}(t)=\mathbf{A}_{r}^{v}+\sum_{s=1}^{S_{c}} \mathbf{A}_{r \tau_{s}} e^{\frac{-t}{\tau_{s}}}
$$

where $\mathbf{A}_{r}^{v}$ denotes the strain concentration tensors for the purely viscous behavior. Tensors $\mathbf{A}_{r \tau_{s}}$ satisfy $\mathbf{A}_{r}^{v}+\sum_{s=1}^{Q} \mathbf{A}_{r \tau_{s}}=\mathbf{A}_{r}^{e}$ with $\mathbf{A}_{r}^{e}$ the average strain concentration tensor for the purely elastic behavior. Relation (9b) thus gives

$$
\begin{aligned}
\tilde{\boldsymbol{\sigma}}_{0}(t)=\left\langle{ }^{\mathrm{T}} \mathbf{A}_{r}^{e}: \boldsymbol{\sigma}_{0 r}(t)+{ }^{\mathrm{T}} \mathbf{A}_{r}^{v}: \sum_{d}\left[\boldsymbol{\sigma}_{0 r}\right]_{d}\right\rangle+ \\
\left\langle\sum_{s}{ }^{\mathrm{T}} \mathbf{A}_{r_{\tau_{s}}}:\left(\sum_{d} e^{\frac{-\left(t-t_{d}\right)}{\tau_{s}}}\left[\boldsymbol{\sigma}_{0 r}\right]_{d}-\boldsymbol{\varrho}_{r \tau_{s}}(t)\right)\right\rangle
\end{aligned}
$$

with $\varrho_{r_{\tau_{s}}}$ the new internal variable verifying

$$
\dot{\boldsymbol{\varrho}}_{r \tau_{s}}(t)+\frac{1}{\tau_{s}} \boldsymbol{\varrho}_{\tau_{\tau_{s}}}(t)=\frac{1}{\tau_{s}} \boldsymbol{\sigma}_{0 r}(t), \quad \boldsymbol{\varrho}_{\tau_{\tau_{s}}}(0)=\mathbf{0}
$$

As for the stress formulation, the integral expression (25) of the thermoviscoelastic constitutive relation has been replaced by an internal variables approach defined by relations (27) and (30).

Detailed equations for the phase average strain are not given here for the sake of conciseness, but they can be obtained using similar developments as those presented above for the stress formulation. Approximating $\mathbf{E}_{r}(t)$ by a Dirichlet series as $\mathbf{D}_{r}(t)$, see equation (21), the expressions obtained for $\langle\varepsilon\rangle_{r}(t)$ and $\left\langle\varepsilon_{\text {res }}\right\rangle_{r}(t)$ have a very similar form to those given above for $\langle\boldsymbol{\sigma}\rangle_{r}(t)$ and $\left\langle\boldsymbol{\sigma}_{\text {res }}\right\rangle_{r}(t)$.

\subsection{Application}

The capabilities of the new formulation with internal variables based on the collocation method are now illustrated for the homogenization problem of a 2-D polycrystal with local anisotropic behavior. Two applications are provided below, the first for prescribed 
overall stress, and the second for prescribed strain. The model has been implemented for both stress and strain formulations in order to compare the relative merit and ease of the numerical implementations. Results will be also compared to the original collocation method.

The chosen microstructure consists of two $(R=2)$ randomly mixed phases, and it is deformed under antiplane shear. The choice of such a simple microstructure aims to obtain a rapid validation of the method, but it is not a limitation. The viscoplastic behavior of similar microstructures has been investigated e.g. in [25, 26, 27]. Owing to this particular microstructure, the Self-Consistent (SC) scheme has been chosen here to solve the symbolic linear thermo-elastic homogenization problem in the LC space. Local elastic and viscous compliances are given by

$$
\mathbf{S}_{r}^{e}=\sum_{k=1}^{2} \frac{1}{4 \mu^{e(k)}} \mathbf{R}_{r}^{(k)} \otimes \mathbf{R}_{r}^{(k)}, \quad \mathbf{S}_{r}^{v}=\sum_{k=1}^{2} \frac{1}{\mu^{v(k)}} \mathbf{R}_{r}^{(k)} \otimes \mathbf{R}_{r}^{(k)}
$$

with $\mu^{e(k)}$ and $\mu^{v(k)}$ the elastic and viscous shear compliances of slip system $(k)$. The following values have been considered for the computations: $\mu^{e(1)}=1 \mathrm{MPa}$, $\mu^{v(1)}=2 \mathrm{MPa} . \mathrm{s}, \mu^{e(2)}=100 \mathrm{MPa}, \mu^{v(2)}=20 \mathrm{MPa}$.s, so that system (2) is stiff compared to system (1). The two mechanical phases are rotated by $90^{\circ}$ from each other, and slip is allowed on two perpendicular slip planes along direction $\mathbf{e}_{3}$ so that the Schmid tensors read $\mathbf{R}_{2}^{(k)}=\frac{1}{2}\left(\mathbf{e}_{k} \otimes \mathbf{e}_{3}+\mathbf{e}_{3} \otimes \mathbf{e}_{k}\right), \mathbf{R}_{1}^{(1)}=\mathbf{R}_{2}^{(2)}$, and $\mathbf{R}_{1}^{(2)}=-\mathbf{R}_{2}^{(1)}$ (see figure 3 ). From the numerical point of view, differential equations appearing in the internal variables formulation have been solved by the Runge-Kutta method. Collocation times $\tau_{s}$ have been distributed on a logarithmic scale between the two extreme relaxation times $\mu^{v(2)} / \mu^{e(2)}$ and $\mu^{v(1)} / \mu^{e(1)}$; numerical applications have been performed for different numbers of collocation times, $5 \leq S_{c} \leq 20$, with no influence on results.

Several macroscopic loadings have been tested. The first case of interest is a creep test $\bar{\sigma}_{13}$ including a stress jump

$$
\left\{\begin{array}{ll}
\bar{\sigma}_{13}=1 \mathrm{MPa} & \text { for } t \leq 2 \mathrm{~s} \\
\bar{\sigma}_{13}=4 \mathrm{MPa} & \text { for } t>2 \mathrm{~s}
\end{array} .\right.
$$

The predicted macroscopic behavior, phase average stress, and phase average strain, are shown in figure 1 . These results have been obtained by means of the original collocation method and the new stress (§3.2) and strain (§3.3) formulations. They are all plotted in figure 1. It can be seen that results obtained with those three formulations are in perfect match with each other at both macroscopic and local levels, which validates the numerical resolution of present developments. It is worth recalling that those three formulation are equivalent, as discussed above. In particular, the stress jumps at $t=0 \mathrm{~s}$ and $t=2 \mathrm{~s}$ and subsequent recovery of both phases are nicely captured.

Another example of challenging test is the response under harmonic loading. We have studied the case of a strain imposed antiplane shear with a constant strain-rate 

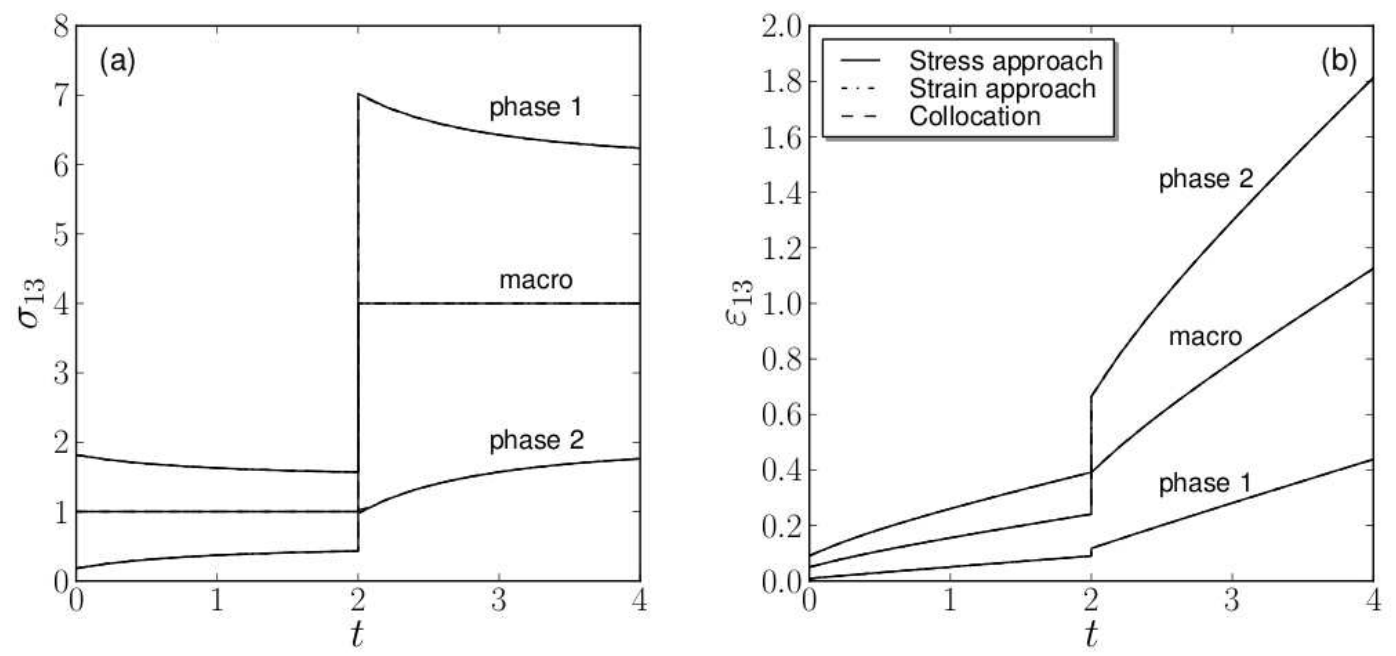

Figure 1. Response of the 2-D polycrystal under the creep loading with stress jump (33). (a) Stress response: 'macro' indicates the prescribed $\bar{\sigma}_{13}(t)$, 'phase 1 ' indicates $\left\langle\sigma_{13}\right\rangle_{1}(t)$, and 'phase 2' $\left\langle\sigma_{13}\right\rangle_{2}(t)$. (b) Corresponding strain response $\left(\bar{\varepsilon}_{13}(t),\left\langle\varepsilon_{13}\right\rangle_{1}(t)\right.$, and $\left.\left\langle\varepsilon_{13}\right\rangle_{2}(t)\right)$. Results from the original collocation method and for both stress and strain approaches are shown.

stage followed by a sinusoidal overall strain stage, as in [5]

$$
\begin{cases}\bar{\varepsilon}_{13}=A \omega t & \text { for } t \leq t_{0} \\ \bar{\varepsilon}_{13}=A \sin \left[\omega\left(t-t_{0}\right)\right] & \text { for } t>t_{0}\end{cases}
$$

with numerical values $A=0.04, \omega=15 \mathrm{~s}^{-1}$, and $t_{0}=1 \mathrm{~s}$. As for the previous example, it is found that the three approaches, namely standard collocation method, stress formulation, and strain formulation provide the same results (figure 2), for the macroscopic behavior but also for phase average stress and strain. At large time, the overall specimen has relaxed from the first loading stage and therefore macroscopic stress and phase average stresses tend to periodic oscillations around 0MPa.

To check the validity of these results, reference solutions were generated with a full-field numerical approach based on Fourier Transforms. The method is described in $[23,28]$ for elastic or viscoplastic composites and polycrystals, and in [29] (with numerical details in [30]) for elasto-viscoplastic behavior. The FFT-based full-field formulation is conceived for periodic unit cells deformed under periodic boundary conditions, and it provides the "exact" (within numerical accuracy) solution of the governing equations. Here, we considered a periodic tile microstructure formed by square grains (see figure 3) which has been found to provide numerical results in very good agreement with theoretical solutions [31] (with which the linear SC scheme also coincides). For linear viscoelastic behaviors, the relaxation spectra of this microstructure exhibits an infinite number of relaxation times. With macroscopic loading (34), the detailed distribution of stress and strain is thus obtained. For the purpose of comparison with mean-field homogenization models, stress and strain fields have been spatially averaged to evaluate 


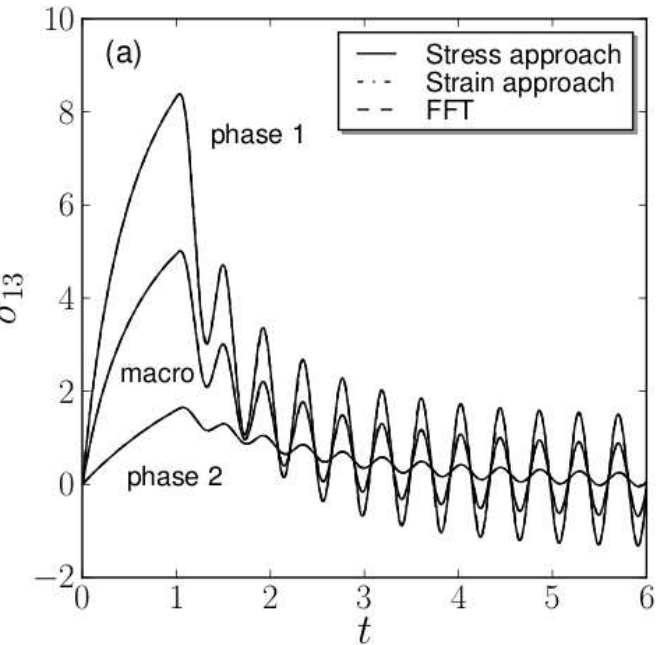

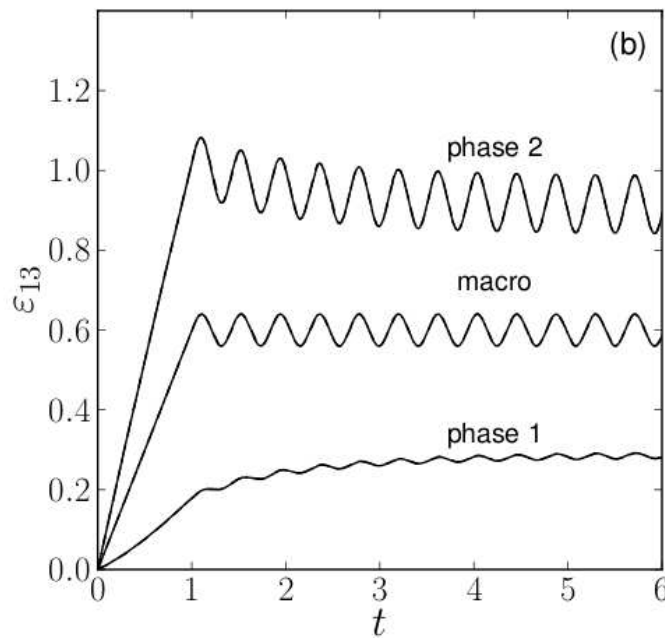

Figure 2. Response of the 2-D polycrystal under the harmonic loading given by (34). Macroscopic and phase average (a) stress and (b) strain responses, as in figure 1. Results from the original collocation method and from both stress and strain approaches are shown, together with those obtained by FFT full-field numerical method.

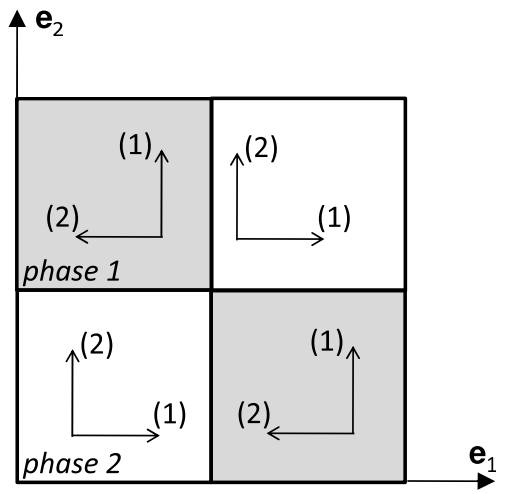

Figure 3. Periodic 2-D microstructure considered for FFT numerical computations. Arrows indicate the normal of slip planes. Slip direction is along $\mathbf{e}_{3}$.

\section{Extension to time- and strain-dependent viscous properties}

The above formulation has been provided for constant elastic $\mathbf{S}_{r}^{e}$ and viscous $\mathbf{S}_{r}^{v}$ compliances. An important consequence of this feature is that coefficients $\mathbf{S}_{\tau_{s}}$ in (12) 
have to be determined only once, independently of the macroscopic prescribed loading. We are now extending the formulation to situations for which this is no more the case. For illustrative purpose, we are considering the case of local constitutive relations including isotropic and kinematic hardening, the latter being time-dependent due to static relaxation mechanisms.

\subsection{Local constitutive behavior law including hardening and revovery}

We consider a local constitutive relation (proposed in [30]) with constant elastic properties, but with a viscous part depending on strain due to isotropic and kinematic hardening and on time due to static relaxation. We consider Voce-type law for isotropic hardening and a simple saturating expression for kinematic hardening as in [32]. The complete constitutive relation reads (spatial position $\mathbf{x}$ has been omitted)

$$
\begin{aligned}
& \dot{\boldsymbol{\varepsilon}}=\mathbf{S}_{r}^{e}: \dot{\boldsymbol{\sigma}}+\dot{\boldsymbol{\varepsilon}}^{v} \\
& \dot{\boldsymbol{\varepsilon}}^{v}=\sum_{k=1}^{K} \dot{\gamma}^{(k)} \mathbf{R}_{r}^{(k)} \\
& \dot{\gamma}^{(k)}=\dot{\gamma}_{0} \frac{\tau^{(k)}-X^{(k)}}{\tau_{0}^{(k)}} \\
& \tau^{(k)}=\mathbf{R}_{r}^{(k)}: \boldsymbol{\sigma} \\
& \dot{\tau}_{0}^{(k)}=\left(\tau_{\text {sta }}^{(k)}-\tau_{0}^{(k)}\right) \sum_{l=1}^{K} H^{(k, l)}\left|\dot{\gamma}^{(l)}\right| \\
& \dot{X}^{(k)}=c \dot{\gamma}^{(k)}-d X^{(k)}\left|\dot{\gamma}^{(k)}\right|-e\left|X^{(k)}\right|^{m} \operatorname{sign}\left(X^{(k)}\right)
\end{aligned}
$$

with $\dot{\varepsilon}^{v}$ the local viscous strain-rate, $\dot{\gamma}^{(k)}$ the shear-rate on slip system $(k), \tau^{(k)}$ the resolved shear stress on that system, and $\dot{\gamma}_{0}, c, d$, and $e$ constant coefficients. With this law, the reference shear stress $\tau_{0}^{(k)}$ for system $(k)$ evolves from an initial value to a saturation value $\tau_{s t a}^{(k)}$ due to isotropic hardening, $H$ being the (constant) interaction matrix between slip systems. Kinematic hardening is due to the backstress $X^{(k)}$ that includes static recovery (coefficient $e$ ). This viscoelastic behavior can be also written

$$
\dot{\varepsilon}=\mathbf{S}_{r}^{e}: \dot{\boldsymbol{\sigma}}+\mathbf{S}^{v}: \boldsymbol{\sigma}+\dot{\boldsymbol{\varepsilon}}_{0}
$$

with

$$
\mathbf{S}^{v}=\sum_{k=1}^{K} \dot{\gamma}_{0}^{(k)} \frac{\mathbf{R}_{r}^{(k)} \otimes \mathbf{R}_{r}^{(k)}}{\tau_{0}^{(k)}}, \quad \dot{\boldsymbol{\varepsilon}}_{0}=-\sum_{k=1}^{K} \dot{\gamma}_{0}^{(k)} \frac{X^{(k)} \mathbf{R}_{r}^{(k)}}{\tau_{0}^{(k)}} .
$$

It is worth noting that the above constitutive relation is defined at any point $\mathbf{x}$ within the polycrystal. Therefore, $\dot{\tau}_{0}^{(k)}(\mathbf{x})$ and $\dot{X}^{(k)}(\mathbf{x})$ should be heterogeneous within each mechanical phase due to the intraphase heterogeneity of $\dot{\gamma}^{(k)}(\mathbf{x})$. A consequence of this is that the compliance $\mathbf{S}^{v}(\mathbf{x})$ and the stress-free strain $\dot{\varepsilon}_{0}(\mathbf{x})$ are also heterogeneous within phases, but then standard homogenization techniques do not apply. To circumvent this difficulty, we have replaced $\dot{\gamma}^{(k)}(\mathbf{x})$ in equations (39) and (40) by its phase average value $\left\langle\dot{\gamma}^{(k)}\right\rangle_{r}$ so that, starting with phase uniform $\dot{\tau}_{0}^{(k)}$ and $\dot{X}^{(k)}$ (now denoted $\dot{\tau}_{0 r}^{(k)}$ and $\dot{X}_{r}^{(k)}$ ), 
$\mathbf{S}^{v}$ and $\dot{\varepsilon}_{0}$ remain phase uniform (denoted $\mathbf{S}_{r}^{v}$ and $\dot{\varepsilon}_{0 r}$ ) so that mean-field homogenization can be carried out. The phase average behavior thus reads

$$
\langle\dot{\boldsymbol{\varepsilon}}\rangle_{r}=\mathbf{S}_{r}^{e}:\langle\dot{\boldsymbol{\sigma}}\rangle_{r}+\mathbf{S}_{r}^{v}:\langle\boldsymbol{\sigma}\rangle_{r}+\dot{\varepsilon}_{0 r} .
$$

The consequence of this approximation will be discussed below.

\subsection{The Incremental Collocation Method (ICM)}

In section 3, both stress and strain approaches have been treated and applied simultaneously, and we have shown that both provide identical results. In the following, for the sake of clarity, only the stress formulation is presented (but we have checked that the strain approach still provides equivalent results) and stress jumps are not included.

The main issue comes from the evolution of the viscous local behavior with time and strain. As a consequence, the coefficients $\mathbf{S}_{\tau_{s}}$ and the homogenized viscous compliance $\tilde{\mathbf{S}}^{v}$ evolve so that the homogenization procedure cannot be applied the same way as previously. This issue can be solved with an incremental resolution, assuming that coefficients $\mathbf{S}_{\tau_{s}}$ are constant during a sufficiently small time increment. Then, equation (14) becomes

$$
\Delta \overline{\boldsymbol{\varepsilon}}=\tilde{\mathbf{S}}^{e}: \Delta \overline{\boldsymbol{\sigma}}+\left.\tilde{\mathbf{S}}^{v}\right|_{1 / 2}: \Delta \boldsymbol{\xi}+\left.\sum_{s=1}^{S} \mathbf{S}_{\tau_{s}}\right|_{1 / 2}: \Delta \boldsymbol{\beta}_{\tau_{s}}+\Delta \tilde{\varepsilon}_{0}
$$

with $\Delta$ denoting the increment between times $t_{n}$ and $t_{n+1}$, e.g. $\Delta \bar{\varepsilon}=\bar{\varepsilon}\left(t_{n+1}\right)-\bar{\varepsilon}\left(t_{n}\right)$. In (44), values for $\mathbf{S}_{\tau_{s}}$ and $\tilde{\mathbf{S}}^{v}$ are taken for half the time increment, e.g. $\left.\mathbf{S}_{\tau_{s}}\right|_{1 / 2}=$ $\left(\mathbf{S}_{\tau_{s}}\left(t_{n}\right)+\mathbf{S}_{\tau_{s}}\left(t_{n+1}\right)\right) / 2$. The evolution laws for $\boldsymbol{\xi}$ and $\boldsymbol{\beta}_{\tau_{s}}$ are the same as in section 3 , see eq.(16). Similarly, the macroscopic thermoelastic strain given in equation (19) is computed using

$$
\Delta \widetilde{\boldsymbol{\varepsilon}}_{0}=\left\langle{ }^{\mathrm{T}} \mathbf{B}_{r}^{e}: \Delta \boldsymbol{\varepsilon}_{0 r}-\left.\sum_{s}{ }^{\mathrm{T}} \mathbf{B}_{r \tau_{s}}\right|_{1 / 2}: \Delta \boldsymbol{\eta}_{r \tau_{s}}\right\rangle
$$

and the phase average stress

$$
\begin{gathered}
\Delta\langle\boldsymbol{\sigma}\rangle_{r}=\mathbf{B}_{r}^{e}: \Delta \overline{\boldsymbol{\sigma}}-\left.\sum_{s} \mathbf{B}_{r_{\tau_{s}}}\right|_{1 / 2}: \Delta \boldsymbol{\beta}_{\tau_{s}}+\mathbf{D}_{r}^{e}:\left(\Delta \widetilde{\boldsymbol{\varepsilon}}_{0}-\Delta \boldsymbol{\varepsilon}_{0 r}\right)- \\
\left.\sum_{s} \mathbf{D}_{r \tau_{s}}\right|_{1 / 2}:\left(\Delta \boldsymbol{\theta}_{\tau_{s}}-\Delta \boldsymbol{\eta}_{r \tau_{s}}\right)
\end{gathered}
$$

where the new macroscopic internal variable $\boldsymbol{\theta}_{\tau_{s}}=\boldsymbol{\lambda}_{r \tau_{s}}+\boldsymbol{\eta}_{r_{\tau_{s}}}$ is introduced for numerical purpose (see Appendix B)

$$
\dot{\boldsymbol{\theta}}_{\tau_{s}}(t)+\frac{1}{\tau_{s}} \boldsymbol{\theta}_{\tau_{s}}(t)=\frac{1}{\tau_{s}} \widetilde{\boldsymbol{\varepsilon}}_{0}(t), \quad \boldsymbol{\theta}_{\tau_{s}}(0)=\mathbf{0} .
$$

Phase average strain increments can then be computed with (41). Note that if hardening is discarded ( $c=d=e=H=0$ ), behaviors given by (44) and (14) are strictly equivalent.

When used with the original integral approach, the standard collocation method applied to polycrystals with local behavior (35-40) requires calculation of coefficients 
$\boldsymbol{S}_{\tau_{s}}$ at each time step. This is also the case for the proposed incremental approach. However, unlike the present formulation, the integral approach requires keeping record of the whole history of $\tilde{\mathbf{S}}$ and $\mathbf{B}_{r}$ from the very first loading stage for the evaluation of integrals (9), which is cumbersome especially when dealing with polycrystals with a large number of mechanical phases and loading steps. With the proposed approach (denoted Incremental Collocation Method, ICM), the numerical resolution is incremental. A stepby-step procedure can be applied, in which the aim of internal variables is to summarize the effects of the whole stress and strain history. This allows studying the polycrystal response for any complex loading with much more ease and flexibility. The algorithm for numerical implementation of the ICM is detailed in Appendix B.

\subsection{Application}

To show the potentiality of the proposed ICM, the microstructure introduced in section 3.4 is investigated for deformation under the complex loading (34). Results are compared with reference solutions generated by the full-field FFT method, as in section 3 . The local behavior (35-40) has been implemented with coefficients indicated in Table 1 and $\dot{\gamma}_{0}=1 \mathrm{~s}^{-1}, m=1$, and $H^{(k, l)}=2 \forall k, l$. Figure 4 shows the effective stress response for two cases: (i) when both isotropic and kinematic are considered (with parameters $c=5 \mathrm{MPa}, d=10, e=0$ ), and (ii) with isotropic hardening only $(c=d=e=0)$. It can be seen that, for both cases, the ICM matches well FFT solutions at the very first loading stage, but then the effective behavior becomes softer than the FFT one, the largest stress discrepancy $\bar{\sigma}_{13}^{\mathrm{FFT}}-\bar{\sigma}_{13}^{\mathrm{INC}}$ being observed close to the stress peak $t \approx 1$ s. At larger time after several loading cycles, the discrepancy decreases until both responses coincide again.

It is recalled that the step-by-step numerical resolution of the ICM provides the same results as the internal variable approach of section 3 when hardening is discarded. Therefore, observed discrepancies are associated with the treatment of hardening. This is now illustrated with the case for which only isotropic hardening has been considered. Here, the main difference with results presented in section 3 is that $\tau_{0}^{(k)}$ is evolving. As discussed above, $\dot{\gamma}^{(k)}(\mathbf{x})$ had to be replaced by $\left\langle\dot{\gamma}^{(k)}\right\rangle_{r}$ in the hardening law for the ICM to be solved with standard mean-field homogenization techniques. Hence, instead of correctly predicting intraphase fluctuations for $\tau_{0}^{(k)}$ as with the FFT approach, the ICM requires phase uniform values. Consequences of this limitation have been investigated in [33] for viscoplastic polycrystals. Here, the ICM underestimates the average value of $\left\langle\tau_{0}^{(k)}\right\rangle_{r}$ compared to FFT reference results. At the same time, the overall behavior for the ICM is softer than for FFT predictions; this can originates from lower $\left\langle\tau_{0}^{(k)}\right\rangle_{r}$ but also from the intraphase heterogeneity of $\tau_{0}^{(k)}$, not predicted by ICM. Figure 5a shows an example of result for slip system $k=1$ of phase $r=1$ (similar trend is observed for other slips systems). Interestingly, the stress discrepancy $\bar{\sigma}_{13}^{\mathrm{FFT}}-\bar{\sigma}_{13}^{\mathrm{INC}}$ is found to be correlated with the standard deviation $\sqrt{\left\langle\tau_{0}^{(k)} \tau_{0}^{(k)}>-<\tau_{0}^{(k)}\right\rangle^{2}}$ (computed with 
Table 1. Parameters of the constitutive relation.

\begin{tabular}{llll}
\hline Slip system $k$ & $\mu_{e}[\mathrm{MPa}]$ & $\tau_{0}(t=0)[\mathrm{MPa}]$ & $\tau_{\text {sta }}[\mathrm{MPa}]$ \\
\hline 1 & 1 & 2 & 4 \\
2 & 100 & 20 & 30 \\
\hline
\end{tabular}

FFT) of $\tau_{0}^{(k)}$. As shown in figure 5b, the highest stress discrepancy at stress peak coincides with the largest standard deviation. Then, as the number of loading cycles increases, $\left\langle\tau_{0}^{(k)}\right\rangle_{r}$ becomes closer to the stationary value and at the same time $\tau_{0}^{(k)}$ becomes more uniform within phases. At large time, ICM and FFT predictions coincide again.
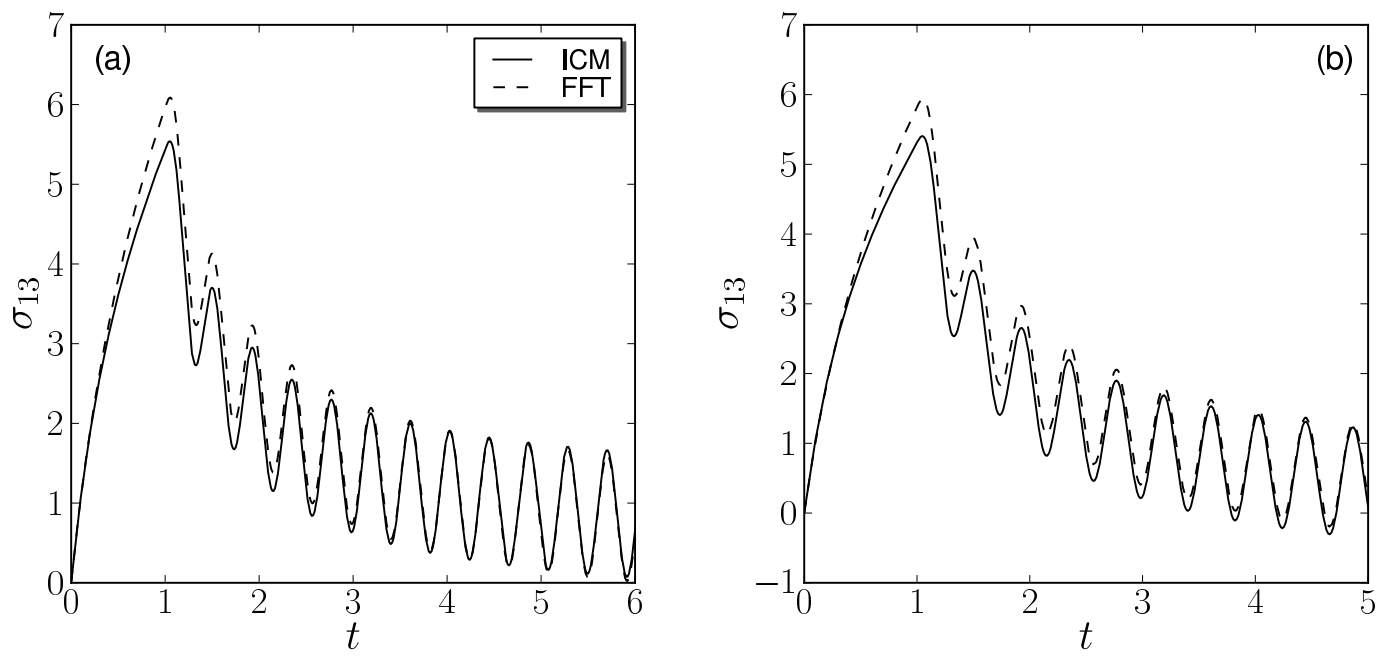

Figure 4. Viscoelastic homogenization with (a) both isotropic and kinematic hardening and (b) isotropic hardening only. Comparisons between incremental collocation and FFT methods for the macroscopic stress response $\bar{\sigma}_{13}$.

\section{Concluding remarks}

In the present article, the equivalence between the collocation method used to inverse Laplace-Carson transforms and an internal variables formulation has been developed for the case of linear thermo-viscoelastic polycrystalline materials exhibiting general anisotropic properties for local and macroscopic behaviors. The method has been applied to 2-D polycrystals with 2 slip systems per phase, deformed under antiplane shear, for macroscopic loading including stress discontinuities and for complex strain loading including a cyclic stage. It has been shown that the internal variable method yields accurate results that perfectly match the standard collocation method. Excellent agreement has also been obtained with references solutions provided by the full-field FFT numerical scheme. The formulation has been extended to the case of time- and strain- 

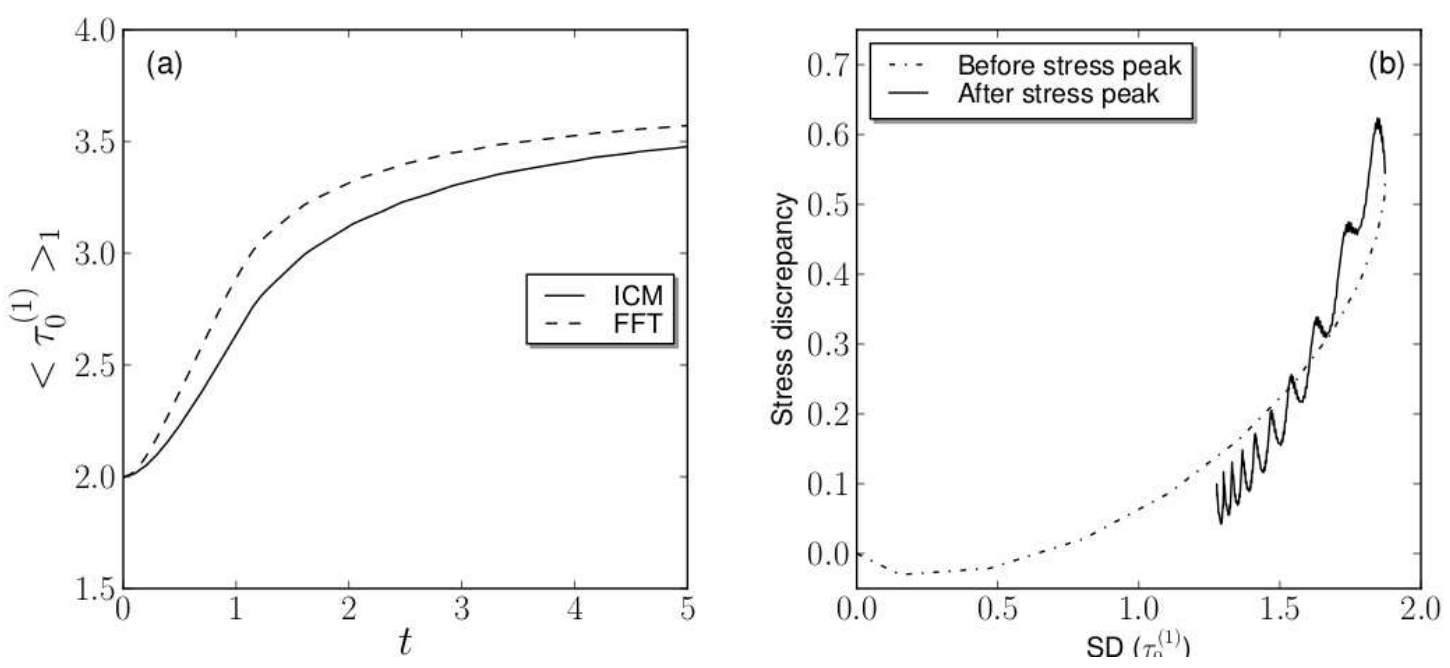

Figure 5. Viscoelastic homogenization with isotropic hardening predicted by the incremental collocation and FFT methods. (a) Evolution of $\left\langle\tau_{0}^{(1)}\right\rangle_{1}$. (b) Difference between the overall stresses $\bar{\sigma}_{13}$ obtained with FFT and ICM as a function of the standard deviation of $\tau_{0}^{(1)}$.

dependent constitutive viscous properties. In that case, an incremental collocation method is proposed. It can be efficiently solved numerically using a step-by-step procedure, and a general algorithm has been proposed. The capability of the method has been illustrated on a 2-D polycrystal, but it is worth noting that solving similar problems with 3-D polycrystals exhibiting few thousand phases requires only a few minutes on a standard laptop. The new method is therefore especially efficient for solving complex loading paths. Moreover, the introduction of isotropic and kinematic hardening at the slip system level has been considered. Some discrepancies with reference FFT results have been observed. They are likely due to the approximation made in considering phase uniform hardening variables within mean-field homogenization. Finally, it is pointed out that the obtained ICM offers a simple framework to address the case of nonlinear behaviors, e.g. using a linearization procedure similar as the one proposed by [21].

\section{Acknowledgments}

This study was partly funded by the French 'Agence Nationale de la Recherche' (project ELVIS, \#ANR-08-BLAN-0138). Authors are very grateful to Renaud Masson (CEACadarache, France) for stimulating discussions on the subject.

\section{Appendix A. Relaxation function for an anisotropic Maxwell behavior}

For general anisotropy, tensors $\mathbf{C}$ and $\mathbf{S}$ verify

$$
\mathrm{C} \star \mathrm{S}=\mathrm{I}, \quad \mathrm{C}^{*}: \mathrm{S}^{*}=\mathbf{I} .
$$


For a Maxwell behaviour, the creep function is $\mathbf{S}(t)=\mathbf{S}^{e}+\mathbf{S}^{v} t$ and the LC transform of the relaxation function thus reads

$$
\mathbf{C}^{*}(p)=\left(\mathbf{S}^{e}+\frac{1}{p} \mathbf{S}^{v}\right)^{-1} .
$$

For general anisotropy, an analytic expression for the relaxation function $\mathbf{C}(t)$, given by the inverse transform of $\mathbf{C}^{*}$, cannot be obtained. However, a closed-form expression can be found for particular symmetry classes by using the spectral decomposition of the fourth-order symmetric tensors [34]. For an isotropic behaviour, local properties read

$$
\mathbf{S}^{e}=\frac{1}{3 k^{e}} \mathbf{J}+\frac{1}{2 \mu^{e}} \mathbf{K}, \quad \mathbf{S}^{v}=\frac{1}{3 k^{v}} \mathbf{J}+\frac{1}{2 \mu^{v}} \mathbf{K}
$$

and thus

$$
\mathbf{C}^{*}(p)=3 k^{e}\left(\frac{\tau_{k} p}{1+\tau_{k} p}\right) \mathbf{J}+2 \mu^{e}\left(\frac{\tau_{\mu} p}{1+\tau_{\mu} p}\right) \mathbf{K}
$$

with $\tau_{k}=k^{v} / k^{e}$ and $\tau_{\mu}=\mu^{v} / \mu^{e}$ two characteristic relaxation times. The corresponding relaxation function is given by

$$
\mathbf{C}(t)=3 k^{e} \exp \left(-t / \tau_{k}\right) \mathbf{J}+2 \mu^{e} \exp \left(-t / \tau_{\mu}\right) \mathbf{K} .
$$

For cubic symmetry, the local properties read

$\mathbf{S}^{e}=\frac{1}{3 k^{e}} \mathbf{J}+\frac{1}{2 \mu_{a}^{e}} \mathbf{K}_{a}+\frac{1}{2 \mu_{b}^{e}} \mathbf{K}_{b}, \quad \mathbf{S}^{v}=\frac{1}{3 k^{v}} \mathbf{J}+\frac{1}{2 \mu_{a}^{v}} \mathbf{K}_{a}+\frac{1}{2 \mu_{b}^{e}} \mathbf{K}_{b}$

and thus

$\mathbf{C}^{*}(p)=3 k^{e}\left(\frac{\tau_{k} p}{1+\tau_{k} p}\right) \mathbf{J}+2 \mu_{a}^{e}\left(\frac{\tau_{\mu_{a}} p}{1+\tau_{\mu_{a}} p}\right) \mathbf{K}_{a}+2 \mu_{b}^{e}\left(\frac{\tau_{\mu_{b}} p}{1+\tau_{\mu_{b}} p}\right) \mathbf{K}_{b}$

with $\tau_{k}=k^{v} / k^{e}, \tau_{\mu_{a}}=\mu_{a}^{v} / \mu_{a}^{e}$ and $\tau_{\mu_{b}}=\mu_{b}^{v} / \mu_{b}^{e}$ three characteristic relaxation times. The corresponding relaxation function thus reads

$\mathbf{C}(t)=3 k^{e} \exp \left(-t / \tau_{k}\right) \mathbf{J}+2 \mu_{a}^{e} \exp \left(-t / \tau_{\mu_{a}}\right) \mathbf{K}_{a}+2 \mu_{b}^{e} \exp \left(-t / \tau_{\mu_{b}}\right) \mathbf{K}_{b}$.

Similar expressions can be obtained for other symmetry classes.

\section{Appendix B. Numerical resolution of the incremental collocation method}

We provide here the algorithm for the step-by-step resolution of the ICM described in section 4 . The case of loadings with prescribed $\overline{\boldsymbol{\sigma}}$ is presented first. Assuming that the homogenization problem has been solved for times $0 \leq t \leq t_{n}$, we seek to find the mechanical response at time $t_{n+1}$ associated to stress and time increments $\Delta \overline{\boldsymbol{\sigma}}$ and $\Delta t$. The algorithm consists essentially of 3 loops, besides the time loop: the outer loop (index $i$ ) for solving $\widetilde{\varepsilon}_{0}$ and $\mathbf{S}^{v}$, the inner loop (index $j$ ) for $\Delta \boldsymbol{\sigma}_{r}$, and a loop for the symbolic homogenization problem.

(1) Computation of $\Delta \boldsymbol{\beta}_{\tau_{s}}$ and $\Delta \boldsymbol{\xi}$ following (16)

(2) Initializations: $\left(\mathbf{S}^{v}\right)_{t_{n+1}}^{i=0}=\left(\mathbf{S}^{v}\right)_{t_{n}} ;\left(\mathbf{S}_{\tau_{s}}\right)_{t_{n+1}}^{i=0}=\left(\mathbf{S}_{\tau_{s}}\right)_{t_{n}} ;\left(\tilde{\mathbf{S}}^{v}\right)_{t_{n+1}}^{i=0}=\left(\tilde{\mathbf{S}}^{v}\right)_{t_{n}} ;\left(\mathbf{B}_{r_{\tau_{s}}}\right)_{t_{n+1}}^{i=0}=$ $\left(\mathbf{B}_{r_{\tau_{s}}}\right)_{t_{n}} ;\left(\mathbf{D}_{r_{\tau_{s}}}\right)_{t_{n+1}}^{i=0}=\left(\mathbf{D}_{r_{\tau_{s}}}\right)_{t_{n}}$ 
(3) Computation of $\Delta\langle\boldsymbol{\sigma}\rangle_{r}$ :

(a) Initialization of $\left(\Delta\langle\boldsymbol{\sigma}\rangle_{r}\right)^{j=0}$

(b) Computation of $(\Delta X)^{j}$ and $\left(\Delta \tau_{0}\right)^{j}$ following (39-40)

(c) Computation of $\left(\Delta \varepsilon_{0 r}\right)^{j}$ and $\left(\Delta\langle\varepsilon\rangle_{r}\right)^{j}$ following (42-41)

(d) Computation of $\left(\Delta \boldsymbol{\eta}_{r_{\tau_{s}}}\right)^{j}$ following (20)

(e) Computation of $\left(\Delta \widetilde{\varepsilon}_{0}\right)^{j}$ following (45)

(f) Computation of $\left(\Delta \boldsymbol{\theta}_{\tau_{s}}\right)^{j}$ following (47)

(g) Actualization of $\left(\Delta\langle\boldsymbol{\sigma}\rangle_{r}\right)^{j+1}$ following (46)

(h) Compute convergence error $\boldsymbol{\delta}_{1}=\left|\left(\Delta\langle\boldsymbol{\sigma}\rangle_{r}\right)^{j+1}-\left(\Delta\langle\boldsymbol{\sigma}\rangle_{r}\right)^{j}\right|$

If $\boldsymbol{\delta}_{1}<$ threshold then go to (4), else $j \leftarrow j+1$ and return to (3.b)

(4) Actualization of $\left(\widetilde{\varepsilon}_{0}\right)_{t_{n+1}}^{i+1}$ and $\left(\mathbf{S}^{v}\right)_{t_{n+1}}^{i+1}$ following (42)

(5) Homogenization of the symbolic thermo-elastic problem $\Rightarrow\left(\mathbf{S}_{\tau_{s}}\right)_{t_{n+1}}^{i+1} ;\left(\tilde{\mathbf{S}}^{v}\right)_{t_{n+1}}^{i+1}$; $\left(\mathbf{B}_{r_{\tau_{s}}}\right)_{t_{n+1}}^{i+1} ;\left(\mathbf{D}_{r_{\tau_{s}}}\right)_{t_{n+1}}^{i+1}$

(6) Compute convergence error $\boldsymbol{\delta}_{2}=\left|\left(\mathbf{S}^{v}\right)_{t_{n+1}}^{i+1}-\left(\mathbf{S}^{v}\right)_{t_{n+1}}^{i}\right|$

If $\boldsymbol{\delta}_{2}<$ threshold then go to (7), else $i \leftarrow i+1$ and return to (3.b)

(7) Output macroscopic and local responses at time $t_{n+1}$, and go to (2) for the next time step

In cases of loading with prescribed $\overline{\boldsymbol{\varepsilon}}$ (instead of $\overline{\boldsymbol{\sigma}}$ as above), the algorithm has to be slightly changed since $\Delta \boldsymbol{\beta}_{\tau_{s}}$ and $\Delta \boldsymbol{\xi}$ cannot be calculated in advance.

(1) Initializations: $\left(\mathbf{S}^{v}\right)_{t_{n+1}}^{i=0}=\left(\mathbf{S}^{v}\right)_{t_{n}} ;\left(\mathbf{S}_{\tau_{s}}\right)_{t_{n+1}}^{i=0}=\left(\mathbf{S}_{\tau_{s}}\right)_{t_{n}} ;\left(\tilde{\mathbf{S}}^{v}\right)_{t_{n+1}}^{i=0}=\left(\tilde{\mathbf{S}}^{v}\right)_{t_{n}} ;\left(\mathbf{B}_{r_{\tau_{s}}}\right)_{t_{n+1}}^{i=0}=$ $\left(\mathbf{B}_{r_{\tau_{s}}}\right)_{t_{n}} ;\left(\mathbf{D}_{r_{\tau_{s}}}\right)_{t_{n+1}}^{i=0}=\left(\mathbf{D}_{r_{\tau_{s}}}\right)_{t_{n}}$

(2) Computation of $\Delta \boldsymbol{\beta}_{\tau_{s}}, \Delta \boldsymbol{\xi}$ and $\tilde{\boldsymbol{\varepsilon}}_{0}$

(a) Initialization : $\left(\Delta \boldsymbol{\beta}_{\tau_{s}}\right)^{j}=\left(\Delta \boldsymbol{\beta}_{\tau_{s}}\right)^{0},(\Delta \boldsymbol{\xi})^{j}=(\Delta \boldsymbol{\xi})^{0}$ and $\left(\Delta \overline{\boldsymbol{\varepsilon}}_{0}\right)^{j}=\left(\Delta \overline{\boldsymbol{\varepsilon}}_{0}\right)^{0}$

(b) Computation of $(\Delta \overline{\boldsymbol{\sigma}})^{j}$ from $(44)$

(c) Computation of $\left(\Delta \boldsymbol{\beta}_{\tau_{s}}\right)^{j+1}$ and $(\Delta \boldsymbol{\xi})^{j+1}$ following (16)

(d) Computation of $\left(\Delta \tilde{\varepsilon}_{0}\right)^{j+1}$

1. Initialization of $\left(\Delta\langle\boldsymbol{\sigma}\rangle_{r}\right)^{k=0}$

2. Computation of $\left(\Delta\langle\boldsymbol{\sigma}\rangle_{r}\right)^{k+1}$ with steps (3.b) to (3.g) above

3. Compute convergence error $\boldsymbol{\delta}_{1}=\left|\left(\Delta\langle\boldsymbol{\sigma}\rangle_{r}\right)^{k+1}-\left(\Delta\langle\boldsymbol{\sigma}\rangle_{r}\right)^{k}\right|$ If $\boldsymbol{\delta}_{1}<$ threshold then $\left(\Delta \tilde{\varepsilon}_{0}\right)^{j+1}=\left(\Delta \tilde{\varepsilon}_{0}\right)^{k}$ and go to (2.e), else $k \leftarrow k+1$ and return to (2.d.2)

(e) Compute convergence error $\boldsymbol{\delta}_{2}$ as the max of normalized value of $\left|\left(\Delta \boldsymbol{\beta}_{\tau_{s}}\right)^{j+1}-\left(\Delta \boldsymbol{\beta}_{\tau_{s}}\right)^{j}\right|,\left|(\Delta \boldsymbol{\xi})^{j+1}-(\Delta \boldsymbol{\xi})^{j}\right|$, and $\left|\left(\Delta \tilde{\boldsymbol{\varepsilon}}_{0}\right)^{j+1}-\left(\Delta \tilde{\boldsymbol{\varepsilon}}_{0}\right)^{j}\right|$

If $\boldsymbol{\delta}_{2}<$ threshold then go to (3), else $j \leftarrow j+1$ and return to (2.b)

(3) Reactualization of $\left(\mathbf{S}^{v s c t}\right)_{t_{n+1}}^{i+1}$ following (??)

(4) Homogenization of the symbolic thermo-elastic problem $\Rightarrow\left(\mathbf{S}_{\tau_{s}}\right)_{t_{n+1}}^{i+1} ; \quad\left(\tilde{\mathbf{S}}^{v}\right)_{t_{n+1}}^{i+1}$; $\left(\mathbf{B}_{r_{\tau_{s}}}\right)_{t_{n+1}}^{i+1} ;\left(\mathbf{D}_{r_{\tau_{s}}}\right)_{t_{n+1}}^{i+1}$

(5) Compute convergence error $\boldsymbol{\delta}_{3}=\left|\left(\mathbf{S}^{v}\right)_{t_{n+1}}^{i+1}-\left(\mathbf{S}^{v}\right)_{t_{n+1}}^{i}\right|$

If $\boldsymbol{\delta}_{3}<$ threshold then go to (6), else $i \leftarrow i+1$ and return to (2) 
(6) Output macroscopic and local responses at time $t_{n+1}$, and go to (2) for the next time step

\section{References}

[1] H. Sabar, M. Berveiller, V. Favier, and S. Berbenni. A new class of micro-macro models for elastic-viscoplastic heterogeneous materials. Int. J. Solids Struct., 39:3257-3276, 2002.

[2] A. Molinari. Averaging models for heterogeneous viscoplastic and elastic viscoplastic materials. J. Eng. Mat. Tech., 124:62-70, 2002.

[3] H. Wang, P. D. Wu, C. N.Tomé, and Y. Huang. A finite strain elastic-viscoplastic self-consistent model for polycrystalline materials. J. Mech. Phys. Solids, 58:594-612, 2010.

[4] M. Coulibaly and H. Sabar. New integral formulation and self-consistent modeling of elasticviscoplastic heterogeneous materials. Int. J. Solids Struct., 48:753-763, 2011.

[5] N. Lahellec and P. Suquet. Effective behavior of linear viscoelastic composites: a time-integration approach. Int. J. Solids Struct., 44:507-529, 2007.

[6] N. Lahellec and P. Suquet. On the effective behavior of nonlinear inelastic composites: I. incremental variational principles. J. Mech. Phys. Solids, 55:1932-1963, 2007.

[7] P. Ponte Castañeda. The effective mechanical properties of nonlinear isotropic composites. $J$. Mech. Phys. Solids, 39:45-71, 1991.

[8] P. Ponte Castañeda and P. Suquet. Nonlinear composites. Adv. Appl. Mech., 34:171-302, 1998.

[9] P. Ponte Castañeda. Second-order homogenization estimates for nonlinear composites incorporating field fluctuations. I - theory. J. Mech. Phys. Solids, 50:737-757, 2002.

[10] J. Mandel. Mécanique des milieux continus. Gauthier-Villars, Paris, France, 1966.

[11] Y. Rougier, C. Stolz, and A. Zaoui. Représentation spectrale en viscoélasticité linéaire des matériaux hétérogènes. C. R. Acad. Sci. Paris, 316:1517-1522, 1993.

[12] S. Beurthey and A. Zaoui. Structural morphology and relaxation spectra of viscoelastic heterogeneous materials. Eur. J. Mech. A/Solids, 19:1-16, 2000.

[13] R. A. Schapery. Approximate methods of transform inversion for viscoelastic stress analysis. In Proc. U.S. Nat. Congr. Appl. Mech. ASME 4th, volume 2, pages 1075-1085, 1962.

[14] P. A. Turner, C. N. Tomé, and C.H. Woo. Self-consistent modelling of nonlinear visco-elastic polycrystals : an approximate scheme. Phil. Mag. A, 70:689-711, 1994.

[15] R. Brenner, R. Masson, O. Castelnau, and A. Zaoui. A "quasi-elastic" affine formulation for the homogenized behaviour of nonlinear viscoelastic polycrystals and composites. Eur. J. Mech. A/Solids, 21:943-960, 2002.

[16] T. L. Cost and E. B. Becker. A multidata method of approximate Laplace transform inversion. Int. J. Num. Meth. Engin., 2:207-219, 1970.

[17] I. Emri and N. W. Tschoegl. Determination of mechanical spectra from experimental responses. Int. J. Solids Struct., 32:817-826, 1995.

[18] R. D. Bradshaw and L. C. Brinson. A sign control method for fitting and interconverting material functions for linearly viscoelastic solids. Mech. Time Dep. Matls., 1:85-108, 1997.

[19] N. Laws and R. McLaughlin. Self-consistent estimates for the viscoelastic creep compliances of composite materials. Proc. R. Soc. Lond., A353:251-273, 1978.

[20] P. A. Turner and C. N. Tomé. Self-consistent modeling of visco-elastic polycrystals : application to irradiation creep and growth. J. Mech. Phys. Solids, 41(7):1191-1211, 1993.

[21] R. Masson and A. Zaoui. Self-consistent estimates for the rate-dependent elastoplastic behaviour of polycrystalline materials. J. Mech. Phys. Solids, 47:1543-1568, 1999.

[22] J. M. Ricaud and R. Masson. Effective properties of linear viscoelastic heterogeneous media: Internal variables formulation and extension to ageing behaviours. Int. J. Solids Struct., 46:1599-1606, 2009.

[23] H. Moulinec and P. Suquet. A fast numerical method for computing the linear and nonlinear mechanical properties of composites. C. R. Acad. Sci. Paris, 318(IIb):0417-1413, 1994. 
[24] A. Rekik and R. Brenner. Optimization of the collocation inversion method for the linear viscoelastic homogenization. Mech. Res. Comm., 38:305-308, 2011.

[25] P. Ponte Castañeda and M. V. Nebozyhn. Variational estimates of the self-consistent type for the effective behaviour of some model nonlinear polycrystals. Proc. R. Soc. Lond., A453:2715-2724, 1997.

[26] G. W. Milton. The theory of composites. Cambridge University Press, 2002.

[27] R. A. Lebensohn, O. Castelnau, R. Brenner, and P. Gilormini. Study of the antiplane deformation of linear 2-d polycrystals with different microstructure. Int. J. Solids Struct., 42:5441-5459, 2005 .

[28] R. A. Lebensohn. N-site modeling of a 3D viscoplastic polycrystal using Fast Fourier Transform. Acta Mater., 49:2723-2737, 2001.

[29] M. I. Idiart, H. Moulinec, P. Ponte Castañeda, and P. Suquet. Macroscopic behavior and field fluctuations in viscoplastic composites: Second-order estimates versus full-field simulations. $J$. Mech. Phys. Solids, 54:1029-1063, 2006.

[30] R. Brenner, O. Castelnau, P. Duval, F. Grennerat, N. Lahellec, M. Montagnat, H. Moulinec, P. Suquet Multi-scale modeling of the mechanical behaviour of polycrystalline ice under transient creep. Procedia IUTAM, to be published.

[31] R. A. Lebensohn, P. Ponte Castañeda, R. Brenner, and O. Castelnau. Full-field vs. homogenization methods to predict microstructure-property relations for polycrystalline materials. In S. Ghosh and D. Dimiduk, editors, Chapter 11 of Computational Methods for Microstructure-Property Relationships, pages 393-441. Springer, 2011.

[32] J. L. Chaboche. A review of some plasticity and viscoplasticity constitutive theories. Int. J. Plast., 24:1642-1693, 2008.

[33] O. Castelnau, R. Brenner, and R.A. Lebensohn. The effect of strain heterogeneity on the workhardening of polycrystals predicted by mean-field approaches. Acta Mater., 54:2745-2756, 2006.

[34] L. J. Walpole. Fourth-rank tensors of the thirty-two crystal classes: Multiplication tables. Proc. R. Soc. Lond., A391:149-179, 1984. 